\title{
ALMOST PERIODIC MILD SOLUTIONS OF A CLASS OF PARTIAL FUNCTIONAL DIFFERENTIAL EQUATIONS
}

\author{
BERND AULBACH AND NGUYEN VAN MINH*
}

AbStract. We study the existence of almost periodic mild solutions of a class of partial functional differential equations via semilinear almost periodic abstract functional differential equations of the form

$$
x^{\prime}=f\left(t, x, x_{t}\right) .
$$

To this end, we first associate with every almost periodic semilinear equation

$$
x^{\prime}=F(t, x)
$$

a nonlinear semigroup in the space of almost periodic functions. We then give sufficient conditions (in terms of the accretiveness of the generator of this semigroup) for the existence of almost periodic mild solutions of $(* *)$ as fixed points of the semigroup. Those results are then carried over to equation $(*)$. The main results are stated under accretiveness conditions of the function $f$ in terms of $x$ and Lipschitz conditions with respect to $x_{t}$.

\section{INTRODUCTION}

In this paper we are mainly concerned with the existence of almost periodic semilinear evolution equations of the form

$$
\frac{d x}{d t}=A x+f\left(t, x, x_{t}\right)
$$

where $A$ is the infinitesimal generator of a $C_{0}$-semigroup $T(t), t \geq 0$ and $f$ is an everywhere defined continuous operator from $\mathbf{R} \times \mathbf{X} \times C$ to $\mathbf{X}$. Throughout this paper we will denote by $C=C_{u}((-\infty, 0], \mathbf{X})$ the space of all uniformly continuous and bounded functions from $(-\infty, 0]$ to $\mathbf{X}$, by $\mathbf{X}$ a

1991 Mathematics Subject Classification. Primary: 34K30, 35R10, 35B15; Secondary: $47 \mathrm{H} 20$.

Key words and phrases. Almost periodic solutions, partial functional differential equations, semilinear equations, semigroups of nonlinear operators, accretiveness.

* Partially supported by the Alexander von Humboldt Foundation. The assistance of the Foundation is gratefully acknowledged.

Received: May 10, 1998. 
given Banach space and by $x_{t}$ the map $x(t+\theta)=x_{t}(\theta), \theta \in(-\infty, 0]$, where $x(\cdot)$ is defined on $(-\infty, a]$ for some $a>0$.

As is well known (see e.g. [2], [5], [10], [12], [18-19], [24-27], [37] and the references therein) many problems on partial differential equations can be stated in the setting of abstract functional differential equations of the form (1). In passing we mention that the case $A=0$ of equation (1) has been treated in [13-17], [28], [31]. In these papers the proofs of the main results are based on an existence theorem for bounded solutions due to Medvedev [20].

Recently, increasing interest in semilinear evolution equations can be observed (see e.g. [12], [18-19], [25-27], [32] and the references therein). This interest arises from a need to extend well-known results on ordinary differential equations to a class of partial differential equations. In this context we consider the existence of almost periodic mild solutions of equation (1). At this point we want to emphasize that our approach to the problem is somewhat different from that used in the papers [13-17], [28], [31]. In fact, we first associate with an equation without delay (equation (2) below) a semigroup of nonlinear operators which then plays a role similar to that of monodromy operator for equations with periodic coefficients. Then we use the results thus obtained in order to study equation (1). Our method, in the case $A=0$, requires a condition on $f$ which is somewhat stronger than that used in the previous papers in order to guarantee the existence of the associated semigroups. However, our method in turn allows to impose accretiveness conditions on the function $f$ in a more general context which seems to be more suitable for equations whose right-hand side $f$ depends explicitly on $t$.

\section{Almost Periodic Solutions of Differential Equations Without Delay}

In this section we deal with almost periodic mild solutions to the semilinear evolution equation

$$
\frac{d x}{d t}=A x+f(t, x), \quad x \in \mathbf{X}
$$

where $\mathbf{X}$ is a Banach space, $A$ is the infinitesimal generator of a $C_{0}$-semigroup $S(t), t \geq 0$ of linear operators of type $\omega$, i.e.

$$
\|S(t) x-S(t) y\| \leq e^{\omega t}\|x-y\|, \forall t \geq 0, x, y \in \mathbf{X},
$$

and $\mathrm{B}$ is an everywhere defined continuous operator from $\mathbf{R} \times \mathbf{X}$ to $\mathbf{X}$. Hereafter, by a mild solution $x(t), t \in[s, \tau]$ of equation (2) we mean a continuous solution of the integral equation

$$
x(t)=S(t-s) x+\int_{s}^{t} S(t-\xi) B(\xi, x(\xi)) d \xi, \forall s \leq t \leq \tau .
$$

A strong solution (for the definition see [34], e.g.) of equation (2) is necessarily a mild solution of this equation. The inverse assertion, however, is not true for a general Banach space $\mathbf{X}$ (see [34] for a counter-example). On the 
other hand, under additional assumptions on $\mathbf{X}$ the mild solutions may be strong solutions. For more information on semilinear equations of the form (2) we refer to [12], [19], [24-25], [34].

Before proceeding we recall some notions and results which will be frequently used later on. We define the bracket $[\cdot, \cdot]$ in a Banach space $\mathbf{Y}$ as follows (see [5], [19])

$$
[x, y]=\lim _{h \rightarrow+0} \frac{\|x+h y\|-\|y\|}{h}=\inf _{h>0} \frac{\|x+h y\|-\|y\|}{h}
$$

Definition 1. Suppose that $F$ is a given operator in a Banach space $\mathbf{Y}$. Then $(F+\gamma I)$ is said to be accretive if and only if for every $\lambda>0$ one of the following equivalent conditions is satisfied

i) $(1-\lambda \gamma)\|x-y\| \leq\|x-y+\lambda(F x-F y)\|, \forall x, y \in D(F)$,

ii) $[x-y, F x-F y] \geq-\gamma\|x-y\|, \forall x, y \in D(F)$.

In particular, if $\gamma=0$, then $F$ is said to be accretive.

Remark. From this definition we may conclude that $(F+\gamma I)$ is accretive if and only if

$$
\|x-y\| \leq\|x-y+\lambda(F x-F y)\|+\lambda \gamma\|x-y\|
$$

for all $x, y \in D(F), \lambda>0,1 \geq \lambda \gamma$.

Definition 2. (Condition H1). Equation (2) is said to satisfy condition H1 if

i) $A$ is the infinitesimal generator of a linear semigroup $\mathrm{S}(\mathrm{t}), t \geq 0$ of type $\omega$ in $\mathbf{X}$,

ii) $B$ is an everywhere defined continuous operator from $\mathbf{R} \times \mathbf{X}$ to $\mathbf{X}$,

iii) For every fixed $t \in \mathbf{R}$, the operator $(-B(t, \cdot)+\gamma I)$ is accretive in $\mathbf{X}$.

The following well-known results are quoted for the reader's convenience (see [12], [19], [34]).

Theorem 1. Let equation (2) satisfy condition $H 1$. Then for every fixed $s \in$ $\mathbf{R}$ and $x \in \mathbf{X}$ there exists a unique mild solution $x(\cdot)$ of equation (2) defined on $[s,+\infty)$. Moreover, if $B$ is independent of $t$, then the mild solutions of equation (2) give rise to a semigroup of nonlinear operators $T(t), t \geq 0$ having the following properties:

$$
\begin{aligned}
& \text { i) } \quad T(t) x=S(t) x+\int_{0}^{t} S(t-\xi) B T^{\xi} x d \xi, \forall t \geq 0, x \in \mathbf{X}, \\
& \text { ii) } \quad\|T(t) x-T(t) y\| \leq e^{(\omega+\gamma) t}\|x-y\|, \forall t \geq 0, x, y \in \mathbf{X} .
\end{aligned}
$$

Throughout this paper we shall denote by $A P(\mathbf{X})$ the space of almost periodic $\mathbf{X}$-valued functions in Bohr's sense with supremum norm, i.e. the space of continuous functions $f$ from $\mathbf{R}$ to $\mathbf{X}$ such that the set $\{f(\cdot+s)$ : $\mathbf{R} \rightarrow \mathbf{X} \mid s \in \mathbf{R}\}$ is precompact in the space of functions $\{f: \mathbf{R} \rightarrow \mathbf{X} \mid$ $f$ bounded and continuous $\}$ equipped with the supremum norm.

The following condition will be used frequently: 
Definition 3. (Condition H2). Equation (2) is said to satisfy condition H2 if for every $u \in A P(\mathbf{X})$ the function $B(\cdot, u(\cdot))$ belongs to $A P(\mathbf{X})$ and if the operator $B_{*}$ taking $u$ into $B(\cdot, u(\cdot))$ is continuous.

The main point of our study is to associate with equation (2) an evolution semigroup which plays a role similar to that of the monodromy operator for equations with periodic cofficients. Hereafter we will denote by $U(t, s)$, $t \geq s$, the evolution operator corresponding to equation (2) which satisfies the assumptions of Theorem 1, i.e. $U(t, s) x$ is the unique solution of equation (3).

Proposition 1. Let the conditions $H 1$ and $H 2$ be satisfied. Then with equation (2) one can associate an evolution semigroup $T^{h}, h \geq 0$ acting on $A P(\mathbf{X})$, defined as

$$
\left[T^{h} v\right](t)=U(t, t-h) v(t-h), \forall h \geq 0, t \in \mathbf{R}, v \in A P(\mathbf{X}) .
$$

Moreover, this semigroup has the following properties:

i) $T^{h}, h \geq 0$ is strongly continuous, and

$$
T^{h} u=S^{h} u+\int_{0}^{h} S^{h-\xi} B_{*}\left(T^{\xi} u\right) d \xi, \forall h \geq 0, u \in A P(\mathbf{X}),
$$

where $\left(S^{h} u\right)(t)=S(h) u(t-h), \forall h \geq 0, t \in \mathbf{R}, u \in A P(\mathbf{X})$.

ii)

$$
\left\|T^{h} u-T^{h} v\right\| \leq e^{(\omega+\gamma) h}\|u-v\|, \quad \forall h \geq 0, u, v \in A P(\mathbf{X}) .
$$

Proof. We first look at the solutions to the equation

$$
w(t)=S^{t-a} z+\int_{a}^{t} S^{t-\xi} B_{*}(w(\xi)) d \xi \forall z \in A P(\mathbf{X}), t \geq a \in \mathbf{R} .
$$

It may be noted that $S^{h}, h \geq 0$ is a strongly continuous semigroup of linear oparators in $A P(\mathbf{X})$ of type $\omega$. Furthermore, for $\lambda>0, \lambda \gamma<1$ and $u, v \in$ $A P(\mathbf{X})$, from the accretiveness of the operators $-B(t, \cdot)+\gamma I$ we get

$$
\begin{aligned}
(1-\lambda \gamma)\|x-y\| & =(1-\lambda \gamma) \sup _{t}\|u(t)-v(t)\| \\
& =\sup _{t}(1-\lambda \gamma)\|u(t)-v(t)\| \\
& \leq \sup _{t}\|u(t)-v(t)-\lambda[B(t, u(t))-B(t, v(t))]\| \\
& =\left\|u-v-\lambda\left(B_{*} u-B_{*} v\right)\right\| .
\end{aligned}
$$

This shows that $\left(-B_{*}+\gamma I\right)$ is accretive. In virtue of Theorem 1 there exists a semigroup $T^{h}, h \geq 0$ such that

$$
\begin{gathered}
T^{h} u=S^{h} u+\int_{0}^{h} S^{h-\xi} B_{*} T^{\xi} u d \xi \\
\left\|T^{h} u-T^{h} v\right\| \leq e^{(\omega+\gamma) h}\|u-v\|, \quad \forall h \geq 0, u, v \in A P(\mathbf{X}) .
\end{gathered}
$$

From this we get

$$
\left[T^{h} u\right](t)=\left[S^{h} u\right](t)+\int_{0}^{h}\left[S^{h-\xi} B_{*}\left(T^{\xi} u\right)\right](t) d \xi, \forall t \in \mathbf{R} .
$$


Thus

$$
\begin{aligned}
{\left[T^{h} u\right](t) } & =S(h) u(t-h)+\int_{0}^{h} S(h-\xi)\left[B_{*}\left(T^{\xi} u\right](t-h+\xi) d \xi\right. \\
& =S(h) u(t-h)+\int_{0}^{h} S(h-\xi) B\left(t+\xi-h,\left[T^{u}\right](t+\xi-h)\right) d \xi \\
& =S(h) u(t-h)+\int_{t-h}^{t} S(t-\eta) B\left(\eta,\left[T^{\eta-(t-h)} u\right](\eta) d \eta .\right.
\end{aligned}
$$

If we denote $\left[T^{t-s} u\right](t)$ by $x(t)$, we get

$$
x(t)=S(t-s) z+\int_{s}^{t} S(t-\xi) B(\xi, x(\xi)) d \xi, \forall t \geq s,
$$

where $z=u(s)$. Consequently, from the uniqueness of mild solutions of equation (2) we get $\left[T^{t-s} u\right](t)=x(t)=U(t, s) u(s)$ and $\left[T^{h} u\right](t)=U(t, t-$ $h) u(t-h)$ for all $t \geq s, u \in A P(Q)$. This completes the proof of the proposition.

The main idea underlying our approach is the following assertion.

Corollary 1. Let all assumptions of Proposition 1 be satisfied. Then a mild solution $x(t)$ of equation (1), defined on the whole real line $\mathbf{R}$, is almost periodic if and only if it is a common fixed point of the evolution semigroup $T^{h}, h \geq 0$ defined in Proposition 1 above.

Proof. Suppose that $x(t)$, defined on the real line $\mathbf{R}$, is an almost periodic mild solution of equation (2). Then from the uniqueness of mild solutions we get

$$
x(t)=U(t, t-h) x(t-h)=\left[T^{h} x\right](t), \forall t \in \mathbf{R} .
$$

This shows that $x$ is a fixed point of $T^{h}$ for every $h>0$. Conversely, suppose that $y(\cdot)$ is any common fixed point of $T^{h}, h \geq 0$. Then

$$
y(t)=\left[T^{t-s} y\right](t)=U(t, s) y(s), \forall t \geq s .
$$

This shows that $y(\cdot)$ is a mild solution of equation (2).

We now apply Corollary 1 in order to get sufficient conditions for the existence of almost periodic mild solutions of equation (2).

Corollary 2. Let all conditions of Proposition 1 be satisfied. Furthermore, let $\omega+\mu$ be negative and $-B_{*}-\mu I$ be accretive. Then there exists a unique almost periodic mild solution of equation (2).

Proof. It is obvious that there exists a unique common fixed point of the semigroup $T^{h}, h \geq 0$. The assertion now follows from Corollary 1 .

Remark. A particular case in which we can check the accretiveness of $-B_{*}-\mu I$ is $\omega+\gamma<0$. In fact, this follows easily from the above estimates for $\|u-v\|$ (see the estimate (8)). 
Another "hyperbolic" case in which there exists a unique common fixed point for the semigroup $T^{h}, h \geq 0$ can be described as follows.

Definition 4. A semigroup $S(t), t \geq 0$ of linear operators in a Banach space $\mathbf{X}$ is said to be hyperbolic if there exist positive constants $K, \alpha$ and a bounded projection $P$ of $\mathbf{X}$ with the following properties:

i) $P S(t)=S(t) P, \forall t \geq 0$,

ii) $(I-P) S(t)(I-P)$ is a homeomorphism of $\operatorname{Ker} P, \forall t \geq 0$,

iii) $\left\|[(I-P) S(t)(I-P)]^{-1}\right\| \leq K e^{-\alpha t}$ and $\|P S(t) P\| \leq K e^{\alpha t}, \forall t \geq 0$.

Corollary 3. Let all conditions of Proposition 1 be satisfied. Moreover, let $A$ be the infinitesimal generator of a hyperbolic semigroup and let $B(t, x)$ satisfy the estimate

$$
\|B(t, x)-B(t, y)\| \leq \delta\|x-y\|, \forall t \in \mathbf{R}, x, y \in \mathbf{X} .
$$

Then, for $\delta$ sufficiently small, equation (2) has a unique almost periodic mild solution.

Proof. It may be seen that

$\|U(t, s) x-U(t, s) y\| \leq e^{\omega(t-s)}\|x-y\|+\int_{s}^{t} e^{\omega(t-\xi)} \delta\|U(\xi, s) x-U(\xi, s) y\| d \xi$.

Using Gronwall's inequality we get

$$
\|U(t, s) x-U(t, s) y\| \leq e^{(\omega+\delta)(t-s)}\|x-y\|, \forall t \geq s, x, y \in \mathbf{X} .
$$

Consequently,

$$
\begin{aligned}
& \|(U(t, s) x-S(t-s) x)-(U(t, s) y-S(t-s) y)\| \leq \\
& \leq \delta \int_{s}^{t} e^{\omega(t-\xi)}\|U(\xi, s) x-U(\xi, s) y\| d \xi \leq \\
& \leq \delta \int_{s}^{t} e^{\omega(t-\xi)} e^{(\omega+\delta)(\xi-s)} d \xi\|x-y\|= \\
& =\delta(t-s) e^{t-s}\|x-y\|, \forall t \geq s, x, y \in \mathbf{X} .
\end{aligned}
$$

Applying this in order to estimate the Lipschitz constant of $T^{1}-S^{1}$ we get

$$
\begin{aligned}
& \left\|\left(T^{1}-S^{1}\right) u-\left(T^{1}-S^{1}\right) v\right\|= \\
& =\sup _{t} \|[U(t, t-1) u(t-1)-S(1) u(t-1)]- \\
& \quad-[U(t, t-1) v(t-1)-S(1) v(t-1) \| \leq \\
& \leq \sup _{t} \delta e^{\omega}\|u(t)-v(t)\| \leq \delta\|u-v\|, \forall u, v \in A P(\mathbf{X}) .
\end{aligned}
$$

Since $S^{1}$ is hyperbolic, 1 does not belong to the spectrum $\sigma\left(S^{1}\right)$. Consequently, applying the Inverse Function Theorem for Lipschitz continuous mappings (see e.g. [19, Proposition 2.3, p. 67]) we observe that if $\delta e^{\omega}<$ $\left\|\left(S^{1}-I\right)^{-1}\right\|$, the maping $T^{1}-I$ is invertible and then it has a unique fixed point. Finally, this is a unique common fixed point for the semigroup $T^{h}, h \geq 0$. Now the assertion of the corollary follows from Corollary 1 . 


\section{Almost Periodic Solutions of Differential Equations With Delays}

In this section we apply the reults of the previous section in order to study the existence of almost periodic mild solutions of the equation

$$
\frac{d x}{d t}=A x+f\left(t, x, x_{t}\right)
$$

where $A$ is defined as in Section 2, and $f$ is an everywhere defined continuous mapping from $\mathbf{R} \times \mathbf{X} \times C$ to $\mathbf{X}$. Hereafter we call a continuous function $x(t)$ defined on the real line $\mathbf{R}$ a mild solution of equation (1) if

$$
x(t)=S(t-s) x(s)+\int_{s}^{t} S(t-\xi) f\left(\xi, x(\xi), x_{\xi}\right) d \xi, \forall t \geq s .
$$

We refer the reader to [32] for more information on the existence and uniqueness of mild solutions of equations of the form (1). We should emphasize that our study is concerned only with the existence of almost periodic mild solutions of equation (1), and not with mild solutions in general. Consequently, the conditions guaranteeing the existence and uniqueness of mild solutions of equation (1) as general as in [32] are not supposed to be a priori conditions.

Definition 5. (Condition H3). Equation (1) is said to satisfy Condition H3 if the following is true:

i) For every $g \in A P(\mathbf{X})$ the mapping $F(t, x)=f\left(t, x, g_{t}\right)$ satisfies conditions $H 1$ and $H 2$ with the same constant $\gamma$.

ii) There exists a constant $\mu$ with $\omega-\mu<0$ such that $-\left(\mu I+F_{*}\right)$ is accretive for every $g \in A P(\mathbf{X})$.

iii) $\left[x-y, f(t, x, \phi)-f\left(t, y, \phi^{\prime}\right)\right] \leq \gamma\|x-y\|+\delta\left\|\phi-\phi^{\prime}\right\|, \forall t \in \mathbf{R}, x, y \in$ $\mathbf{X}, \phi, \phi^{\prime} \in C$.

Theorem 2. Let condition H3 hold. Then for $\delta$ sufficiently small (see the estimate (13) below), equation (1) has an almost periodic mild solution.

Proof. First we fix a function $g \in A P(\mathbf{X})$. In view of Proposition 1 we observe that the equation

$$
\frac{d x}{d t}=A x+F(t, x)
$$

has a unique almost periodic mild solution, where $F(t, x)=f\left(t, x, g_{t}\right)$. We denote this solution by $T g$. Thus, we have defined an operator $T$ acting on $A P(\mathbf{X})$. We now prove that $T$ is a strict contraction mapping. In fact, let us denote by $U(t, s)$ and $V(t, s)$ the Cauchy operators

$$
\begin{aligned}
U(t, s) x & =S(t-s) x+\int_{s}^{t} S(t-\xi) f\left(\xi, U(\xi, s) x, g_{\xi}\right) d \xi \\
V(t, s) x & =S(t-s) x+\int_{s}^{t} S(t-\xi) f\left(\xi, V(\xi, s) x, h_{\xi}\right) d \xi
\end{aligned}
$$

for given $g, h \in A P(\mathbf{X}), x \in \mathbf{X}, t \geq s$. 
Putting $u(t)=U(t, s) x, v(t)=V(t, s) x$ for given $s, x$, from the assumptions we have

$$
\left[u(t)-v(t), f\left(t, u(t), g_{t}\right)-f\left(t, v(t), h_{t}\right)\right] \leq m(t,\|u(t)-v(t)\|),
$$

where $m(t,\|u(t)-v(t)\|)=\gamma\|u(t)-v(t)\|+\delta\|h-g\|$. Using this we get

$$
\begin{aligned}
\|u(t)-v(t)\| \leq & \|u(t-\eta)-v(t-\eta)\|+\eta m(t,\|u(t)-v(t)\|)+ \\
& +\int_{t-\eta}^{t}\left\|S(t-\xi) f\left(\xi, u(\xi), h_{\xi}\right)-f\left(t, u(t), h_{t}\right)\right\| d \xi+ \\
& +\int_{t-\eta}^{t}\left\|S(t-\xi) f\left(\xi, v(\xi), g_{\xi}\right)-f\left(t, v(t), g_{t}\right)\right\| d \xi .
\end{aligned}
$$

Now let us fix arbitrary real numbers $a \leq b$. Since the functions $S(t-$ $\xi) f\left(\xi, u(\xi), h_{\xi}\right)$ and $S(t-\xi) f\left(\xi, v(\xi), g_{\xi}\right)$ are uniformly continuous on the set $a \leq \xi \leq t \leq b$, for every $\epsilon>0$ there exists an $\eta_{0}=\eta_{0}(\epsilon)$ such that

$$
\begin{aligned}
\left\|S(t-\xi) f\left(\xi, u(\xi), h_{\xi}\right)-f\left(t, u(t), h_{t}\right)\right\| & <\epsilon, \\
\left\|S(t-\xi) f\left(\xi, v(\xi), g_{\xi}\right)-f\left(t, v(t), g_{t}\right)\right\| & <\epsilon,
\end{aligned}
$$

for all $\|t-\xi\|<\eta_{0}$ and $t \leq \xi \in[a, b]$. Hence, denoting $\|u(t)-v(t)\|$ by $\alpha(t)$, for $\eta<\eta_{0}$ we have

$$
\alpha(t)-e^{\omega \eta} \alpha(t-\eta) \leq \eta m(t, \alpha(t))+2 \eta \epsilon .
$$

Applying this estimate repeatedly, we get

$$
\alpha(t)-e^{\omega(t-s)} \leq \sum_{i=1}^{n} e^{\omega\left(t-t_{i}\right)} m\left(t_{i}, \alpha\left(t_{i}\right)\right) \Delta_{i}+2 \epsilon \sum_{i=1}^{n} e^{\omega\left(t-t_{i}\right)} \Delta_{i},
$$

where $t_{0}=s<t_{1}<t_{2}<\ldots<t_{n}=t$ and $\left|t_{i}-t_{i-1}\right|=\Delta_{i}$. Thus, since $\epsilon$ is arbitrary, and since the function $m$ is continuous, we get

$$
\begin{aligned}
\alpha(t)-e^{\omega(t-s)} \alpha(s) & \leq \int_{s}^{t} e^{\omega(t-\xi)} m(\xi, \alpha(\xi)) d \xi \\
& =\int_{s}^{t} e^{\omega(t-\xi)}(\gamma \alpha(\xi)+\delta\|h-g\|) d \xi
\end{aligned}
$$

Applying Gronwall's inequality we get

$$
\alpha(t) \leq e^{(\gamma+\omega)(t-s)} \alpha(s)+e^{\gamma(t-s)+\omega t}\left(\frac{e^{-\omega s}-e^{-\omega t}}{\omega}\right) \delta\|h-g\| .
$$

Because of the identity $\alpha(s)=\|u(s)-v(s)\|=\|U(s, s) x-V(s, s) x\|=0$, from the above estimate we obtain

$$
\sup _{t-1 \leq \xi \leq t}\|U(\xi, t-1) x-V(\xi, t-1) x\| \leq \frac{e^{\gamma+\omega}-e^{\gamma}}{\omega} \delta\|h-g\| .
$$

Now let us denote by $T_{h}^{t}, T_{g}^{t}, t \geq 0$ the respective evolution semigroups corresponding to equations (10) and (11). Since $T h$ and $T g$ are defined as the 
unique fixed points $u_{0}, v_{0}$ of $T_{h}^{1}, T_{g}^{1}$, respectively, we have

$$
\begin{aligned}
\|T h-T g\|=\left\|u_{0}-v_{0}\right\|= & \left\|T_{h}^{1} u_{0}-T_{g}^{1} v_{0}\right\| \leq\left\|T_{h}^{1} u_{0}-T_{g}^{1} u_{0}\right\|+ \\
& +\left\|T_{g}^{1} u_{0}-T_{g}^{1} v_{0}\right\| \\
\leq & \frac{e^{\gamma+\omega}-e^{\gamma}}{\omega} \delta\|h-g\|+e^{\omega-\mu}\left\|u_{0}-v_{0}\right\| \\
= & N \delta\|h-g\|+e^{\omega-\mu}\|T h-T g\|,
\end{aligned}
$$

where $N=\left(e^{\gamma+\omega}-e^{\gamma}\right) / \omega$. Finally, we have

$$
\|T h-T g\| \leq \frac{e^{\gamma}\left(e^{\omega}-1\right)}{\omega\left(1-e^{\omega-\mu}\right)} .
$$

Thus, if the estimate

$$
\delta<\frac{\omega\left(1-e^{\omega-\mu}\right)}{e^{\gamma}\left(e^{\omega}-1\right)}
$$

holds true, then $T$ is a strict contraction mapping in $A P(\mathbf{X})$. By virtue of the Contraction Mapping Principle $T$ has a unique fixed point. It is easy to see that this fixed point is an almost periodic mild solution of equation (1). This completes the proof of the theorem.

Remarks. 1) In case $\omega=0, \gamma=-\mu$ we get the estimate

$$
\delta<e^{\mu}-1=\mu+\mu^{2} / 2+\ldots
$$

which guarantees the existence of the fixed point of $T$.

2) If $\omega+\gamma<0$, then we can choose $\mu=-\gamma$, and therefore we get the accretiveness condition on $-\left(F_{*}+\mu I\right)$. However, in general, the condition $\omega+\gamma<0$ is a very strong restriction on the coefficients of equation (1), if $f$ depends explicitly on $t$.

\section{EXAMPLES}

In applications one frequently encounters functions $f$ from $\mathbf{R} \times \mathbf{X} \times C \rightarrow \mathbf{X}$ of the form

$$
f\left(t, x, g_{t}\right)=F(t, x)+G\left(t, g_{t}\right), \forall t \in \mathbf{R}, x \in \mathbf{X}, g_{t} \in C,
$$

where $F$ satisfies condition ii) of Definition 5 and $G(t, y)$ is Lipschitz continuous with respect to $y \in C$, i.e.

$$
\|G(t, y)-G(t, z)\| \leq \delta\|y-z\|, \forall t \in \mathbf{R}, y, z \in C
$$

for some positive constant $\delta$. With $f$ in this form numerous examples of partial functional differential equations fitting into our abstract framework can be found (see e.g. [2], [18], [19], [26], [27], in particular the recent book by $\mathrm{Wu}[35])$.

In order to describe a concrete example we consider a bounded domain $\Omega$ in $\mathbf{R}^{n}$ with smooth boundary $\partial \Omega$ and suppose that

$$
A(x, D) u=\sum_{|\alpha| \leq 2 m} a_{\alpha}(x) D^{\alpha} u
$$


is a strongly elliptic differential operator in $\Omega$. Then, defining the operator

$$
A u=A(x, D) u, \forall u \in D(A)=W^{2 m, 2}(\Omega) \cap W_{0}^{m, 2}(\Omega)
$$

we know from [27, Theorem 3.6] that the operator $-A$ is the infinitesimal generator of an analytic semigroup of contractions on $L^{2}(\Omega)$. Now let $f, g$ : $\mathbf{R} \times \Omega \times \mathbf{R} \rightarrow \mathbf{R}$ be Lipschiz continuous and define the operators $F(t, w)(x)=$ $f(t, x, w(x))$ and $G(t, w)(x)=g(t, x, w(x))$ where $t \in \mathbf{R}, x \in \Omega$ and $w \in$ $L^{2}(\Omega)$. Then, for any positive constant $r$, the boundary value problem

$$
\begin{aligned}
\frac{\partial u(t, x)}{\partial t} & =A(x, D) u(t, x)+f(t, x, u(t, x))+g(t, x, u(t-r, x)) \text { in } \Omega, \\
u(t, x) & =0 \text { on } \partial \Omega
\end{aligned}
$$

fits into the abstract setting of equation (1).

Acknowledgement. This work has been carried out while the second author (N.V.M.) was a fellow of the Alexander von Humboldt Foundation. The assistance of the Foundation is gratefully acknowledged. N.V.M. thanks Professors B. Aulbach, R. Nagel, F. Räbiger and the Division of Functional Analysis of the Institute of Mathematics, University of Tübingen, for hospitality, useful discussions and encouragement. N.V.M. also thanks Professor G. Webb for useful suggestions and encouragement. Finally, the authors thank the referee for useful comments leading to an improvement of the original version of this paper.

\section{References}

[1] B. Aulbach and Nguyen Van Minh, Bounded and almost periodic solutions and evolution semigroups associated with nonautonomous functional differential equations, submitted.

[2] V. Barbu, Nonlinear semigroups and differential equations in Banach spaces, Noordhoff, Groningen, 1976.

[3] H. Brézis and A. Pazy, Convergence and approximation of semigroups of nonlinear operators in Banach spaces, J. Func. Anal. 9 (1972), 63-74.

[4] H. Brézis, Operateurs maximaux monotones et semigroupes de contractions dans les espaces de Hilbert, North-Holland, Amsterdam, 1973.

[5] M. G. Crandall, Nonlinear semigroups and evolution governed by accretive operators, Proc. Sympos. Pure Math., Amer. Math. Soc., 45 (1986), Part 1, 305-337.

[6] M. G. Crandall and T. M. Liggett, Generation of nonlinear transformations on general Banach spaces, Amer. J. Math. 93 (1971), 265-298. 
[7] Ju. L. Daleckii and M. G. Krein, Stability of Solutions of Differential Equations in Banach Space, Transl. Math. Monographs, 43, Amer. Math. Soc., Providence, R.I., 1974.

[8] A. M. Fink, Almost Periodic Differential Equations, Lect. Notes in Math. 377, Springer, Berlin, 1974.

[9] J. K. Hale, Asymptotic behavior of dissipative systems, Mathematical Surveys and Monographs, 25, Amer. Math. Soc., Providence, R.I., 1988.

[10] A. Haraux, Nonlinear evolution equations - global behaviour of solutions, Lect. Notes in Math. 841, Springer, Berlin 1981.

[11] Y. Hino, S. Murakami and T. Naito, Functional Differential Equations with Infinite Delay, Lect. Notes in Math. 1473, Springer, Berlin, 1991.

[12] T. Iwamiya, Global existence of mild solutions to semilinear differential equations in Banach spaces, Hiroshima Math. J. 16 (1986), 499-530.

[13] A. G. Kartsatos, Almost periodic solutions to nonlinear systems, Boll. Un. Mat. Ital. 9 (1974), 10-15.

[14] S. Kato, Almost periodic solutions of functional differential equations with infinite delays in a Banach space, Hokkaido Math. J. 23 (1994), 465-474.

[15] S. Kato, Some remarks on nonlinear ordinary differential equations in a Banach space, Nonlinear Anal. 5 (1981), 81-93.

[16] S. Kato and M. Imai, Remarks on the existence of almost periodic solutions of systems of nonlinear differential equations, Nonlinear Anal. 25 (1995), 409-415.

[17] S. Kato and M. Imai, On the existence of periodic and almost periodic solutions for nonlinear systems, Nonlinear Anal. 24 (1995), 1183-1192.

[18] H. Komatsu, (ed.), Functional Analysis and Related Topics, 1991, Lect. Notes in Math. 1540, Springer, Berlin, 1993.

[19] R. Martin, Nonlinear operators and differential equations in Banach spaces, Wiley-Interscience, New York, 1976.

[20] N. V. Medvedev, Certain tests for the existence of bounded solutions of systems of differential equations, Differentsial'nye Uravneniya, 4 (1968), 1258-1264.

[21] R. Nagel, (ed.), One-parameter Semigroups of Positive Operators, Lect. Notes in Math. 1184, Springer, Berlin, 1984.

[22] Nguyen Van Minh, Nonlinear semigroups and nonlinear nonautonomous differential equations, Dokl. Belorussian Acad. Sci., 37 (1993), 113-117. (in Russian)

[23] Nguyen Van Minh, Semigroups and stability of nonautonomous differential equations in Banach spaces, Trans. Amer. Math. Soc. 345 (1994), 223-242.

[24] S. Oharu and T. Takahashi, Locally Lipschitz continuous perturbations of linear dissipative operators and nonlinear semigroups, Proc. Amer. Math. Soc. 100 (1987), 187-194.

[25] S. Oharu and T. Takahashi, Characterization of nonlinear semigroups associated with semilinear evolution equations, Trans. Amer. Math. Soc. 311 (1989), 593-619. 
[26] N. H. Pavel, Nonlinear evolution operators and semigroups, applications to partial differential equations, Lect. Notes in Math. 1260, Springer, Berlin, 1987.

[27] A. Pazy, "Semigroups of linear operators and applications to partial differential equations", Appl. Math. Sci. 44, Springer, Berlin, 1983.

[28] G. Seifert, Almost periodic solutions for a certain class of almost periodic systems, Proc. Amer. Math. Soc. 84 (1982), 47-51.

[29] G. Seifert, Nonlinear evolution equations with almost periodic time dependence, SIAM J. Math. Anal. 18 (1987), 387-392.

[30] G. Seifert, Almost periodicity in semiflows, Proc. Amer. Math. Soc. 123 (1995), 2895-2899.

[31] G. Seifert, Almost periodic solutions for delay-differential equations with infinite delays, J. Differential Equations, 41(1981), 416-425.

[32] J. S. Shin, Comparison theorems and uniqueness of mild solutions to semilinear functional differential equations in Banach spaces, Nonlinear Anal. 23 (1994), 825-847.

[33] C. C. Travis and G. F. Webb, Existence and stability for partial functional differential equations, Trans. Amer. Math. Soc. 200 (1974), 395418.

[34] G. F. Webb, Continuous nonlinear perturbations of linear accretive operators in Banach spaces, J. Func. Anal. 10 (1972), 191-203.

[35] J. Wu, Theory and Applications of Partial Functional Differential Equations, Applied Math. 119, Springer, Berlin, 1996.

[36] T. Yoshizawa, Stability Theory and the Existence of Periodic Solutions and Almost Periodic Systems, Appl. Math. 14, Springer, Berlin, 1975.

[37] K. Yosida, Functional Analysis, Springer, Berlin, 1965.

Department of Mathematics

University of Augsburg

D-86135 Augsburg, GERMANY

E-mail address: aulbach@math.uni-augsburg.de

Department of Mathematics

The University of Electro-Communications

Chofugaoka 1-5-1, CHOFu

TOKYO 182, JAPAN

E-mail address: minh@im.uec.ac.jp 


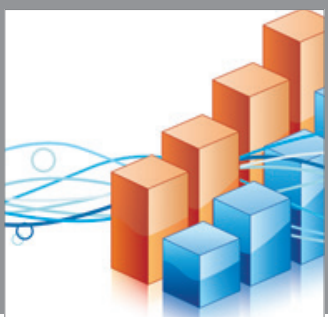

Advances in

Operations Research

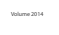

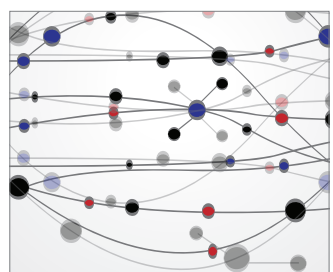

\section{The Scientific} World Journal
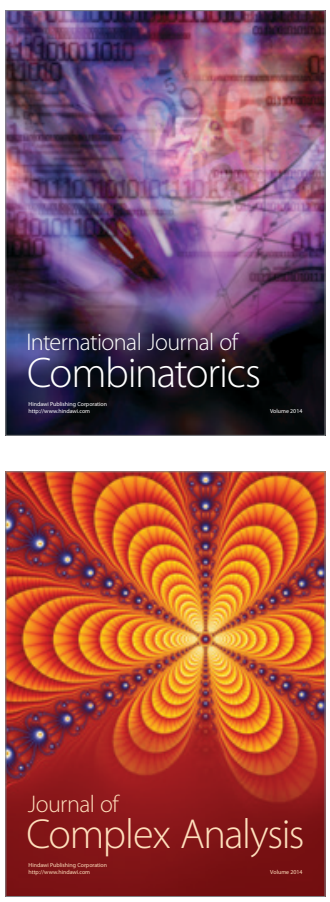

International Journal of

Mathematics and

Mathematical

Sciences
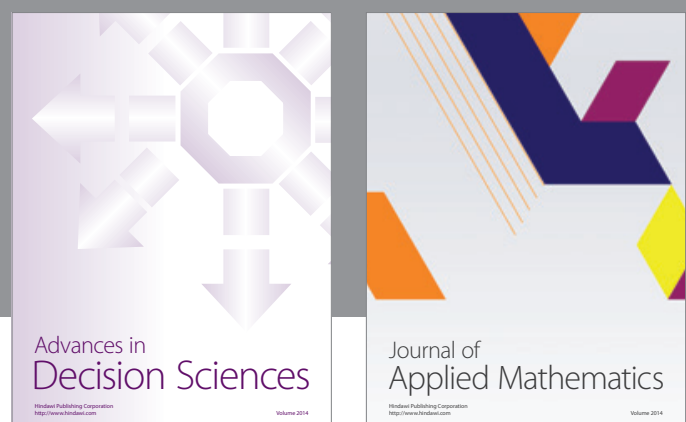

Journal of

Applied Mathematics
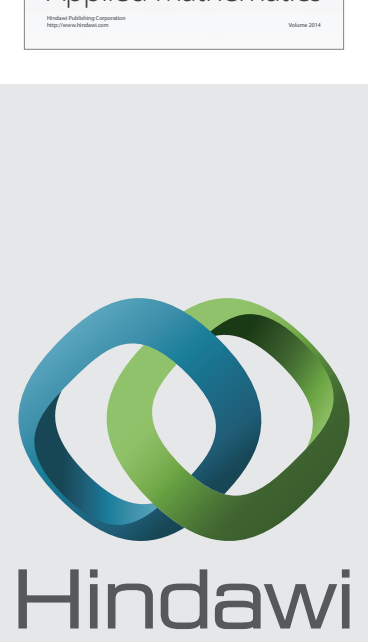

Submit your manuscripts at http://www.hindawi.com
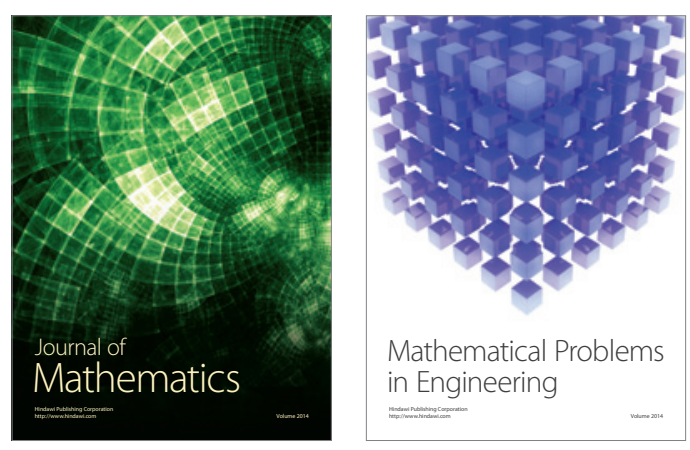

Mathematical Problems in Engineering
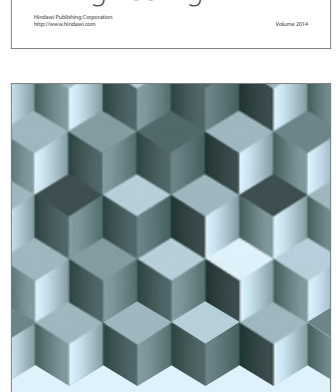

Journal of

Function Spaces
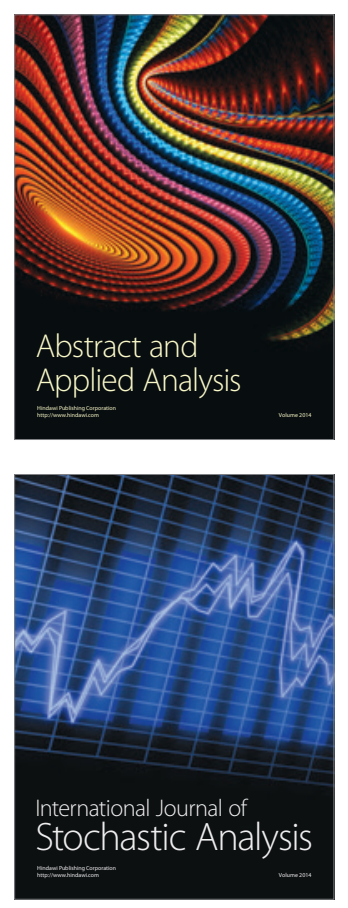

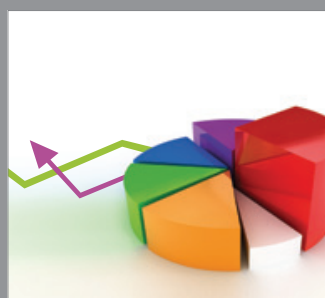

ournal of

Probability and Statistics

Promensencen
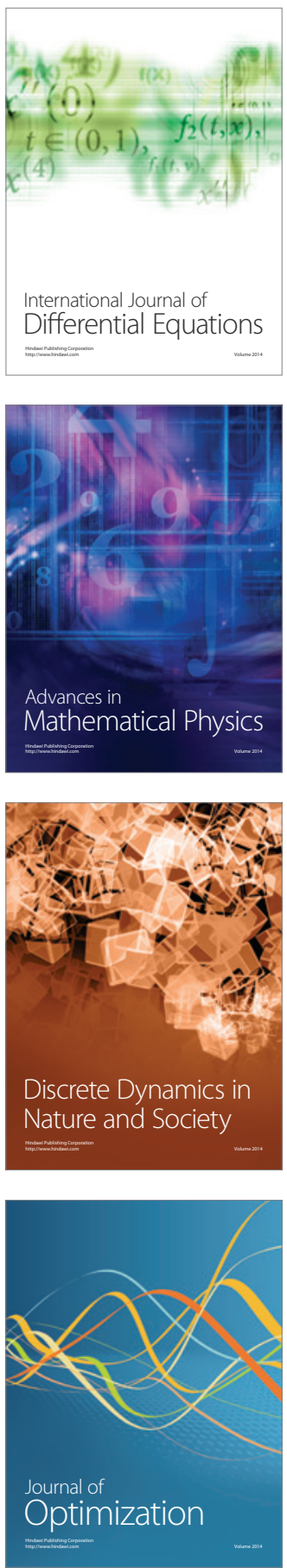